\title{
APPLICATIONS OF A THEOREM CONCERNING SETS WITH CONNECTED SECTIONS
}

\author{
BiAgio RicCERI
}

Dedicated to Professor Ky Fan, with my greatest admiration and esteem

As the reader can notice, the title of the present paper differs from that of [3] only because the term connected replaces the term convex. This is not casual. Indeed, it remains our aim to show, by means of a series of further applications, the usefulness of our recent Theorem 2.3 of [6] which, in a certain sense, can be regarded as a "connected" version of the famous Theorems 1 ' and 2 of [3].

In the sequel, given a product space $X \times Y$, we denote by $p_{X}$ and $p_{Y}$ the projections from $X \times Y$ onto $X$ and $Y$, respectively. Moreover, if $A \subseteq X \times Y$, then for every $x \in X$ and $y \in Y$, we put

$$
A_{x}=\{v \in Y:(x, v) \in A\} \quad \text { and } \quad A^{y}=\{u \in X:(u, y) \in A\} .
$$

Also, when, in proper settings, they will appear, the symbols $\bar{B}$, $\operatorname{int}(B), \partial B$, $\operatorname{aff}(B)$, and $\operatorname{ri}(B)$ will denote, respectively, the closure, the interior, the boundary, the affine hull, and the relative interior (that is, the interior in $\operatorname{aff}(B))$ of the set $B$.

For the reader's convenience, we recall the statement of Theorem 2.3 of [6]:

Theorem 1 ([6], Theorem 2.3). Let $X, Y$ be two topological spaces, with $Y$ admitting a continuous bijection onto $[0,1]$, and let $S, T$ be two subsets of $X \times Y$, with $S$ connected and, for each $x \in X, T_{x}$ connected. Moreover, assume

1991 Mathematics Subject Classification. 54D05, 54F45, 34A60, 47H17, 90A14. 
that either $T^{y}$ is open for each $y \in Y$, or $Y$ is compact and $T$ is closed. Then at least one of the following assertions holds:

$(\alpha) p_{X}(T) \neq X$.

(ß) $p_{Y}(S) \neq Y$ and $\left\{y \in Y:\left(p_{X}(S) \times\{y\}\right) \cap T=\emptyset\right\} \neq \emptyset$.

$(\gamma) S \cap T \neq \emptyset$.

Let us also recall the following result which is useful to recognize the connectedness of a given set in a product space.

Proposition 1 ([6], Theorem 2.4). Let $X, Y$ be two topological spaces and let $S$ be a subset of $X \times Y$. Assume that at least one of the following four sets of conditions is satisfied:

$\left(\gamma_{1}\right) p_{Y}(S)$ is connected, $S^{y}$ is connected for each $y \in Y$, and $S_{x}$ is open for each $x \in X$;

$\left(\gamma_{2}\right) p_{Y}(S)$ is connected, $X$ is compact, $S$ is closed, and $S^{y}$ is connected for each $y \in Y$;

$\left(\gamma_{3}\right) p_{X}(S)$ is connected, $S_{x}$ is connected for each $x \in X$, and $S^{y}$ is open for each $y \in Y$;

$\left(\gamma_{4}\right) p_{X}(S)$ is connected, $Y$ is compact, $S$ is closed and $S_{x}$ is connected for each $x \in X$.

Under such hypotheses, $S$ is connected.

Then, thanks to Proposition 1, we have the following particular case of Theorem 1:

Theorem 2 ([6], Theorem 2.5). Let $X, Y$ be two topological spaces, with $Y$ admitting a continuous bijection onto $[0,1]$, and let $S, T$ be two subsets of $X \times Y$. Assume that at least one of the following eight sets of conditions is satisfied:

$\left(\delta_{1}\right) p_{Y}(S)$ is connected, $S^{y}$ is connected for each $y \in Y, S_{x}$ is open for each $x \in X, T_{x}$ is connected for each $x \in X$, and $T^{y}$ is open for each $y \in Y$;

$\left(\delta_{2}\right) p_{Y}(S)$ is connected, $Y$ is compact, $S^{y}$ is connected for each $y \in Y, S_{x}$ is open for each $x \in X, T$ is closed, and $T_{x}$ is connected for each $x \in X$;

$\left(\delta_{3}\right) p_{Y}(S)$ is connected, $X$ is compact, $S$ is closed, $S^{y}$ is connected for each $y \in Y, T_{x}$ is connected for each $x \in X$, and $T^{y}$ is open for each $y \in Y$;

$\left(\delta_{4}\right) p_{Y}(S)$ is connected, $X$ and $Y$ are compact, $S$ and $T$ are closed, $S^{y}$ is connected for each $y \in Y$, and $T_{x}$ is connected for each $x \in X$;

$\left(\delta_{5}\right) p_{X}(S)$ is connected, $S_{x}$ and $T_{x}$ are connected for each $x \in X$, and $S^{y}$ and $T^{y}$ are open for each $y \in Y$; 
$\left(\delta_{6}\right) p_{X}(S)$ is connected, $Y$ is compact, $S_{x}$ is connected for each $x \in X, S^{y}$ is open for each $y \in Y, T$ is closed, and $T_{x}$ is connected for each $x \in X$;

$\left(\delta_{7}\right) p_{X}(S)$ is connected, $Y$ is compact, $S$ is closed, $S_{x}$ and $T_{x}$ are connected for each $x \in X$, and $T^{y}$ is open for each $y \in Y$;

$\left(\delta_{8}\right) p_{X}(S)$ is connected, $Y$ is compact, $S$ and $T$ are closed, and $S_{x}$ and $T_{x}$ are connected for each $x \in X$.

Then at least one of the following assertions holds:

$(\alpha) p_{X}(T) \neq X$.

( $\beta) p_{Y}(S) \neq Y$ and $\left\{y \in Y:\left(p_{X}(S) \times\{y\}\right) \cap T=\emptyset\right\} \neq \emptyset$.

( $\gamma) S \cap T \neq \emptyset$.

Before starting with our series of applications of Theorems 1 and 2, we point out the following

Proposition 2. Let $Y$ be a connected topological space admitting a continuous bijection onto $[0,1]$. Then there are exactly two distinct points $u, v \in Y$ such that the sets $Y \backslash\{u\}$ and $Y \backslash\{v\}$ are connected. Precisely, one has $\{u, v\}=\left\{\varphi^{-1}(0), \varphi^{-1}(1)\right\}$ for any continuous bijection $\varphi: Y \rightarrow[0,1]$.

Proof. Let $\varphi$ be any continuous bijection from $Y$ onto $[0,1]$. Let us show that $Y \backslash\left\{\varphi^{-1}(0)\right\}$ is connected. Arguing by contradiction, assume that there are two non-empty, open, disjoint sets $A, B$ such that $A \cup B=Y \backslash\left\{\varphi^{-1}(0)\right\}$ (note that $Y$ turns out to be Hausdorff). Since $Y \backslash A$ and $Y \backslash B$ are two (not singletons) closed sets whose intersection (that is, $\left\{\varphi^{-1}(0)\right\}$ ) and union (that is, $Y$ ) are connected, it follows that they are connected too ([5], p. 133). Consequently, $\varphi(Y \backslash A)$ and $\varphi(Y \backslash B)$ are two non-degenerate subintervals of $[0,1]$ each of which contains 0 . Of course, this is against the fact that $(Y \backslash A) \cap(Y \backslash B)=\left\{\varphi^{-1}(0)\right\}$. Likewise, it is seen that $Y \backslash\left\{\varphi^{-1}(1)\right\}$ is connected. Now, let $z \in Y \backslash\left\{\varphi^{-1}(0), \varphi^{-1}(1)\right\}$. Then the sets $\varphi^{-1}\left(\left[0, \varphi(z)[)\right.\right.$ and $\left.\left.\varphi^{-1}(] \varphi(z), 1\right]\right)$ are non-empty and open, and their union is $Y \backslash\{z\}$. So, $Y \backslash\{z\}$ is disconnected. This completes the proof. $\square$

The points $u, v$ in the statement of Proposition 2 will be called the extreme points of $Y$.

Now, we start with the following

TheOREM 3. Let $X, Y$ be two topological spaces, with $Y$ connected and admitting a continuous bijection onto $[0,1]$, and let $S$ be a connected subset of $X \times Y$. In addition, assume that either $S^{y}$ is closed for each $y \in Y$, or $S$ is open and $Y$ is compact. Finally, suppose that, for each $x \in X$, the set $Y \backslash S_{x}$ is connected. Then, if $u, v$ are the extreme points of $Y$, at least one of the following assertions holds: 
(a) There exists $x_{0} \in X$ such that $S_{x_{0}}=Y$.

(b) $S^{u}=\emptyset$.

(c) $S^{v}=\emptyset$.

Moreover, if $S^{u}=\emptyset\left(\right.$ resp. $\left.S^{v}=\emptyset\right)$, then $S^{v}=p_{X}(S)\left(\right.$ resp. $\left.S^{u}=p_{X}(S)\right)$.

Proof. Let $\varphi$ be any continuous bijection from $Y$ onto $[0,1]$. By Proposition 2 , we have $\{u, v\}=\left\{\varphi^{-1}(0), \varphi^{-1}(1)\right\}$. For instance, let $u=\varphi^{-1}(0)$ and $v=$ $\varphi^{-1}(1)$. Assume that (b) and (c) do not hold. Then one has $u, v \in p_{Y}(S)$. Hence, since $p_{Y}(S)$ is connected, we have $\varphi\left(p_{Y}(S)\right)=[0,1]$, and so $p_{Y}(S)=Y$. Now, put

$$
T=(X \times Y) \backslash S .
$$

It is seen at once that $S, T$ satisfy the assumptions of Theorem 1 . Consequently, since $(\beta)$ and $(\gamma)$ are violated, $(\alpha)$ (that is, our present (a)) does hold.

Now, assume that $S^{u}=\emptyset$. Let $x \in p_{X}(S)$. Since $Y \backslash S_{x}$ is connected, $[0,1] \backslash$ $\varphi\left(S_{x}\right)$ turns out to be a proper subinterval of $[0,1]$ containing 0 . Consequently, $1 \in \varphi\left(S_{x}\right)$, that is, $v \in S_{x}$, and so $x \in S^{v}$, as desired. The claim with the roles of $u, v$ interchanged is proved in a similar way.

In particular, applying Theorem 3, we get

TheOREM 4. Let $X$ be a compact topological space, $Y \subseteq \mathbb{R}$ an interval, and $S$ a closed subset of $X \times Y$ such that $Y \backslash S_{x}$ is connected for each $x \in X$, and $S^{y}$ is connected for each $y \in Y$. Then either $p_{Y}(S) \neq Y$, or $S_{x_{0}}=Y$ for some $x_{0} \in X$.

Proof. Suppose that $p_{Y}(S)=Y$. Owing to the compactness of $X$, to get our conclusion it suffices to show that the family $\left\{S^{y}\right\}_{y \in Y}$ has the finite intersection property. So, let $y_{1}<y_{2}<\ldots<y_{n}$ be $n$ points in $Y$. Thanks to Proposition 1 (case $\left.\left(\gamma_{2}\right)\right)$, the set $S \cap\left(X \times\left[y_{1}, y_{n}\right]\right)$ is connected. Then, applying Theorem 3 in an obvious way, we get $x^{*} \in X$ such that $\left[y_{1}, y_{n}\right] \subseteq S_{x^{*}}$. Hence, $x^{*} \in \bigcap_{i=1}^{n} S^{y_{i}}$, as desired.

REMARK 1. Theorem 2 is particularly useful when the sections $S^{y}$ are such that after removing suitable subsets from them, they remain connected. In fact, in such a case, generally either we are allowed to require the connectedness of the sections $T_{x}$ only for particular points $x \in X$, or we can bring out some suitable qualitative property of $S \cap T$. We now indicate two specific situations. For the first of them, we need the following

Proposition 3. Let $E$ be a Hausdorff topological vector space, $A \subseteq E$ an infinite-dimensional closed affine manifold, $\Omega \subseteq A$ a convex set whose interior in $A$ is non-empty, and $K \subseteq E$ a relatively compact set. Then the set $\Omega \backslash K$ is connected. 
Proof. We first prove the proposition in the case where $A=E$. Let $x, y \in$ $\operatorname{int}(\Omega) \backslash \bar{K}$. Fix a closed circled neighbourhood $V$ of the origin such that

$$
V+V \subseteq((\operatorname{int}(\Omega) \backslash \bar{K})-x) \cap((\operatorname{int}(\Omega) \backslash \bar{K})-y)
$$

Observe, in particular, that $V$ is connected. Since $E$ is infinite-dimensional, $V$ is not compact. Consequently, there is a net $\left\{y_{\alpha}\right\}$ in $V$ having no cluster point in $E$. We claim that, for some $\alpha$, the segment joining $x$ and $y+y_{\alpha}$ does not meet $\bar{K}$.

On the contrary, assume that, for each $\alpha$, there is $\lambda_{\alpha} \in[0,1]$ such that $\lambda_{\alpha}\left(y+y_{\alpha}\right)+\left(1-\lambda_{\alpha}\right) x \in \bar{K}$. Now, consider a $\delta>0$ such that $\delta(y-x) \in V$. Thanks to our previous choices, it is seen that $\lambda_{\alpha}>\delta$. Since $\bar{K}$ is compact, the net $\left\{\lambda_{\alpha}\left(y+y_{\alpha}\right)+\left(1-\lambda_{\alpha}\right) x\right\}$ admits a subnet, say $\left\{\lambda_{\alpha_{\beta}}\left(y+y_{\alpha_{\beta}}\right)+\left(1-\lambda_{\alpha_{\beta}}\right) x\right\}$, converging to a point $z \in \bar{K}$. On the other hand, also the net $\left\{\lambda_{\alpha_{\beta}}\right\}$ admits a subnet, say $\left\{\lambda_{\alpha_{\beta_{\gamma}}}\right\}$, converging to a point $\lambda \in[\delta, 1]$. Consequently, $z-(1-\lambda) x$ is the limit of $\left\{\lambda_{\alpha_{\beta_{\gamma}}}\left(y+y_{\alpha_{\beta_{\gamma}}}\right)\right\}$. Hence, $\lambda^{-1}(z-(1-\lambda) x)-y$ is the limit of $\left\{y_{\alpha_{\beta_{\gamma}}}\right\}$, and so it is a cluster point of $\left\{y_{\alpha}\right\}$, a contradiction.

Then let $\alpha$ be such that the segment, say $S\left(x, y+y_{\alpha}\right)$, joining $x$ and $y+y_{\alpha}$ does not meet $\bar{K}$. Since $\operatorname{int}(\Omega)$ is convex, we have $S\left(x, y+y_{\alpha}\right) \subseteq \operatorname{int}(\Omega) \backslash \bar{K}$. Therefore, $S\left(x, y+y_{\alpha}\right) \cup(y+V)$ is a connected subset of $\operatorname{int}(\Omega) \backslash \bar{K}$ containing $x$ and $y$. This shows that $\operatorname{int}(\Omega) \backslash \bar{K}$ is connected. Now, taking into account that $\bar{\Omega}=\overline{\operatorname{int}(\Omega)}$, we have

$$
\operatorname{int}(\Omega) \backslash \bar{K} \subseteq \Omega \backslash K \subseteq \overline{\operatorname{int}(\Omega) \backslash \bar{K}}
$$

and so $\Omega \backslash K$ is connected.

Finally, to prove our proposition when $A \neq E$, it suffices to observe that, since $A$ is closed, $K \cap A$ is relatively compact in $A$ and that $A$ is affinely homeomorphic to an infinite-dimensional Hausdorff topological vector space.

We then have

THEOREM 5. Let $X$ be a non-empty set in a Hausdorff topological vector space $E, K$ a relatively compact subset of $E, Y$ a connected topological space admitting a continuous bijection onto $[0,1]$, and $S, T$ two subsets of $X \times Y$. Assume that:

(i) $S^{y}$ is convex, $\operatorname{aff}\left(S^{y}\right)$ is infinite-dimensional and closed in $E, \operatorname{ri}\left(S^{y}\right)$ is non-empty for each $y \in p_{Y}(S)$, and $S_{x}$ is open in $Y$ for each $x \in X \backslash K$;

(ii) $T_{x}$ is connected for each $x \in X \backslash K$;

(iii) either $T^{y} \backslash K$ is open in $X \backslash K$ for each $y \in Y$, or $Y$ is compact and $T \backslash(K \times Y)$ is closed in $(X \backslash K) \times Y$.

Then at least one of the following assertions holds:

(a) $X \backslash\left(K \cup p_{X}(T)\right) \neq \emptyset$. 
(b) $p_{Y}(S) \neq Y$.

(c) For every set $V \subseteq X \times Y$ such that $V^{y}$ is relatively compact in $E$ for each $y \in Y$ and $V_{x}$ is closed in $Y$ for each $x \in X \backslash K$, one has $(S \backslash(V \cup(K \times Y))) \cap T \neq \emptyset$.

Proof. Assume that (a) and (b) do not hold. Let $V$ be as in (c). Then, by Proposition 3, $(S \backslash(V \cup(K \times Y)))^{y}$ is non-empty and connected for each $y \in Y$, and $(S \backslash(V \cup(K \times Y)))_{x}$ is open for each $x \in X \backslash K$. Hence, since $Y$ is connected, the sets $S \backslash(V \cup(K \times Y))$ and $T \backslash(K \times Y)$ satisfy either $\left(\delta_{1}\right)$ or $\left(\delta_{2}\right)$ of Theorem 2, applied taking $(X \backslash K) \times Y$ as product space. So, $(S \backslash(V \cup(K \times Y))) \cap T \neq \emptyset$.

The other situation to which we alluded in Remark 1 involves the covering dimension in $\mathbb{R}^{n}$. So, for each set $A \subseteq \mathbb{R}^{n}$, we denote by $\operatorname{dim}(A)$ its covering dimension ([2], p. 54).

Theorem 6. Let $X \subseteq \mathbb{R}^{n}$ be a non-empty set, $Y$ a connected topological space admitting a continuous bijection onto [0,1], and $S, T$ two subsets of $X \times Y$. Assume that:

(i) $S^{y}$ is connected and open in $\mathbb{R}^{n}$ for each $y \in Y$, and $S_{x}$ is open in $Y$ for each $x \in X$;

(ii) $T_{x}$ is connected for each $x \in X$;

(iii) either $T^{y}$ is open in $X$ for each $y \in Y$, or $Y$ is compact and $T$ is closed in $X \times Y$.

Then at least one of the following assertions holds:

(a) $p_{X}(T) \neq X$.

(b) $p_{Y}(S) \neq Y$.

(c) For every set $V \subseteq X \times Y$ such that $\operatorname{dim}\left(V^{y}\right) \leq n-2$ for each $y \in Y$ and $V_{x}$ is closed in $Y$ for each $x \in X$, one has $(S \backslash V) \cap T \neq \emptyset$.

Proof. The proof goes exactly as that of Theorem 5 , with $K=\emptyset$. The only difference is that, this time, the connectedness of each $(S \backslash V)^{y}$ follows directly from a celebrated theorem of Mazurkiewicz ([2], p. 80).

Proceeding in a way by now evident, we also get

Theorem 7. Let $X, Y$ be as in Theorem 6 , let $S, T \subseteq X \times Y$, and let $K \subseteq X$ be such that $\operatorname{dim}(K) \leq n-2$. Assume that:

(i) $S^{y}$ is connected and open in $\mathbb{R}^{n}$ for each $y \in Y$, and $S_{x}$ is open in $Y$ for each $x \in X \backslash K$;

(ii) $T_{x}$ is connected for each $x \in X \backslash K$;

(iii) either $T^{y} \backslash K$ is open in $X \backslash K$ for each $y \in Y$, or $Y$ is compact and $T \backslash(K \times Y)$ is closed in $(X \backslash K) \times Y$. 
Then at least one of the following assertions holds:

(a) $X \backslash\left(K \cup p_{X}(T)\right) \neq \emptyset$.

(b) $p_{Y}(S) \neq Y$.

(c) $(S \backslash(K \times Y)) \cap T \neq \emptyset$.

Before stating our next result, we need the following

Proposition 4. Let $\Omega \subseteq \mathbb{R}^{n}$ be a non-empty open connected set and $A$, $B$ two proper subsets of $\Omega$, both closed in $\Omega$, such that $\Omega=A \cup B$. Then $\operatorname{dim}(A \cap B) \geq n-1$.

Proof. If $\operatorname{int}(A) \cap \operatorname{int}(B) \neq \emptyset$, clearly one has $\operatorname{dim}(A \cap B)=n([2]$, p. 76). So, let us assume that $\operatorname{int}(A) \cap \operatorname{int}(B)=\emptyset$. Since $A, B$ are closed in $\Omega$, one has

$$
\Omega \backslash(A \cap B) \subseteq \operatorname{int}(A) \cup \operatorname{int}(B) .
$$

On the other hand, since $A, B$ are proper subsets of $\Omega$, both $\operatorname{int}(A)$ and $\operatorname{int}(B)$ meet $\Omega \backslash(A \cap B)$. So, $\Omega \backslash(A \cap B)$ is disconnected. At this point, our conclusion follows directly from the already quoted theorem of Mazurkiewicz.

Now, we are able to establish the following

ThEOREM 8. Let $[a, b]$ be a compact real interval and $T$ a subset of $\mathbb{R}^{n} \times[a, b]$ which is closed in $p_{\mathbb{R}^{n}}(T) \times[a, b]$. Then, for every non-empty connected subset $X$ of $p_{\mathbb{R}^{n}}(T)$ which is open in $\operatorname{aff}(X)$ and such that $T_{x}$ is connected for each $x \in X$, at least one of the following assertions holds:
(a) $X \subseteq T^{a}$.
(b) $X \subseteq T^{b}$.
(c) There exists some $y \in] a, b\left[\right.$ such that $\operatorname{dim}\left(T^{y} \cap X\right) \geq \operatorname{dim}(X)-1$.

Proof. Assume that (a) and (b) do not hold. Put

$$
\Gamma=X \backslash\left(T^{a} \cup T^{b}\right) .
$$

We distinguish two cases.

First, suppose that $\Gamma \neq \emptyset$. Note that $\Gamma$ is open in $\operatorname{aff}(X)$. Now, fix a sequence $\left\{Y_{k}\right\}$ of (non-degenerate) compact subintervals of $] a, b[$ such that $] a, b[$ $=\bigcup_{k \in \mathbb{N}} Y_{k}$. For each $k \in \mathbb{N}$, put $V_{k}=\bigcup_{y \in Y_{k}} T^{y}$. By Theorem 7.1.16 of [4], the set $V_{k}$ is closed in $p_{\mathbb{R}^{n}}(T)$. Clearly, one has $\Gamma \subseteq \bigcup_{k \in \mathbb{N}} V_{k}$. Endowed with the relative topology, $\Gamma$ turns out to be a Baire space. Hence, there is some $k^{*} \in \mathbb{N}$ such that the interior of $V_{k^{*}} \cap \Gamma$ in $\Gamma$, and so in $\operatorname{aff}(X)$, is non-empty. Choose a non-empty connected set $W \subseteq V_{k^{*}} \cap \Gamma$ which is open in $\operatorname{aff}(X)$. We claim that there exists $y_{0} \in Y_{k^{*}}$ such that $\operatorname{dim}\left(T^{y_{0}} \cap W\right) \geq \operatorname{dim}(W)-1$.

Arguing by contradiction, assume that $\operatorname{dim}\left(T^{y} \cap W\right) \leq \operatorname{dim}(W)-2$ for each $y \in Y_{k^{*}}$. Put

$$
S=\left(W \times Y_{k^{*}}\right) \backslash T
$$


Then, thanks to the theorem of Mazurkiewicz, $S^{y}$ is non-empty and connected for each $y \in Y_{k^{*}}$. Consequently, we can apply Theorem 2 (case $\left(\delta_{2}\right)$ ) to the sets $S$ and $T \cap\left(W \times Y_{k^{*}}\right)$, upon taking $W \times Y_{k^{*}}$ as product space. But, recalling the definition of $W$, we see that the conclusion of Theorem 2 does not hold, which is absurd.

So, the claimed $y_{0}$ actually exists. Observing that $W \subseteq X$ and $\operatorname{dim}(W)=$ $\operatorname{dim}(X)$, we then have $\operatorname{dim}\left(T^{y_{0}} \cap X\right) \geq \operatorname{dim}(X)-1$, which yields (c).

Now, suppose that $X \subseteq T^{a} \cup T^{b}$. In other words, $T^{a} \cap X$ and $T^{b} \cap X$ are proper subsets of $X$, both closed in $X$, whose union is $X$. Then, by Proposition 4, we have $\operatorname{dim}\left(T^{a} \cap T^{b} \cap X\right) \geq \operatorname{dim}(X)-1$. But, if $x \in T^{a} \cap T^{b} \cap X$, then since $T_{x}$ is connected, we have $T_{x}=[a, b]$, that is to say, $x \in T^{y}$ for each $y \in[a, b]$. Hence, in the present case, we get $\operatorname{dim}\left(T^{y} \cap X\right) \geq \operatorname{dim}(X)-1$ even for each $y \in[a, b]$. This completes the proof.

REMark 2. In Theorem 8 , the closedness assumption on $T$ cannot be dropped, in general. Indeed, if $T$ is the graph of a bijection from $\mathbb{R}^{2}$ onto $[0,1]$, taking, for instance, $X=\mathbb{R}^{2}$, none of (a), (b), (c) holds.

Here is an application of Theorem 8 to control theory. Let $b$ be a positive real number and let $F$ be a given multifunction from $[0, b] \times \mathbb{R}^{n}$ into $\mathbb{R}^{n}$. We denote by $\mathcal{S}_{F}$ the set of all Carathéodory solutions of the problem $x^{\prime} \in F(t, x), x(0)=0$ in $[0, b]$. That is to say,

$$
\mathcal{S}_{F}=\left\{u \in A C\left([0, b], \mathbb{R}^{n}\right): u^{\prime}(t) \in F(t, u(t)) \text { a.e. in }[0, b], u(0)=0\right\}
$$

where, of course, $A C\left([0, b], \mathbb{R}^{n}\right)$ denotes the space of all absolutely continuous functions from $[0, b]$ into $\mathbb{R}^{n}$. For each $t \in[0, b]$, put

$$
\mathcal{A}_{F}(t)=\left\{u(t): u \in \mathcal{S}_{F}\right\} .
$$

In other words, $\mathcal{A}_{F}(t)$ denotes the attainable set at time $t$. Also, put

$$
V_{F}=\bigcup_{t \in[0, b]} \mathcal{A}_{F}(t)
$$

Finally, set

$$
C_{F}=\left\{x \in \mathbb{R}^{n}:\left\{t \in[0, b]: x \in \mathcal{A}_{F}(t)\right\} \text { is connected }\right\} .
$$

With these notations, we have the following

TheOREM 9. Assume that $F$ has non-empty compact convex values and bounded range. Moreover, assume that $F(\cdot, x)$ is measurable for each $x \in \mathbb{R}^{n}$ and that $F(t, \cdot)$ is upper semicontinuous for a.e. $t \in[0, b]$. Then, for every nonempty connected set $X \subseteq V_{F} \cap C_{F}$ which is open in $\operatorname{aff}(X)$ and different from $\{0\}$, one has the following alternative: either

$$
X \subseteq \mathcal{A}_{F}(b)
$$


or

$$
\operatorname{dim}\left(\mathcal{A}_{F}(t) \cap X\right) \geq \operatorname{dim}(X)-1
$$

for some $t \in] 0, b[$.

Proof. Put

$$
T=\left\{(x, t) \in \mathbb{R}^{n} \times[0, b]: x \in \mathcal{A}_{F}(t)\right\} .
$$

Under our assumptions, by a well-known result (see, for instance, Theorem 7.1 of [1]), the set $T$ turns out to be closed. Now, our conclusion follows directly from Theorem 9, taking into account that $\mathcal{A}_{F}(0)=\{0\}$.

REMARK 3. On the basis of Theorem 9, it would be interesting to investigate the structure of the set $C_{F}$.

The next result, another application of Theorem 2, concerns the existence of Nash equilibrium points.

THEOREM 10. Let $X$ be a Hausdorff compact topological space, $Y$ an arc, and $f, g$ two continuous real functions on $X \times Y$ such that, for each $\lambda \in \mathbb{R}$, $x_{0} \in X, y_{0} \in Y$, the sets $\left\{x \in X: f\left(x, y_{0}\right) \geq \lambda\right\}$ and $\left\{y \in Y: g\left(x_{0}, y\right) \geq \lambda\right\}$ are connected. Then there exists $\left(x^{*}, y^{*}\right) \in X \times Y$ such that

$$
f\left(x^{*}, y^{*}\right)=\max _{x \in X} f\left(x, y^{*}\right) \quad \text { and } \quad g\left(x^{*}, y^{*}\right)=\max _{y \in Y} g\left(x^{*}, y\right) .
$$

Proof. For each $x \in X, y \in Y$, put

$$
\alpha(x)=\max _{v \in Y} g(x, v) \quad \text { and } \quad \beta(y)=\max _{u \in X} f(u, y) .
$$

Next, consider the sets

$$
S=\{(x, y) \in X \times Y: f(x, y)=\beta(y)\}
$$

and

$$
T=\{(x, y) \in X \times Y: g(x, y)=\alpha(x)\} .
$$

The continuity of $f$ and $g$ readily implies that $S$ and $T$ are closed. On the other hand, for each $x \in X, y \in Y$, one has

$$
S^{y}=\bigcap_{n \in \mathbb{N}}\{u \in X: f(u, y) \geq \beta(y)-1 / n\}
$$

and

$$
T_{x}=\bigcap_{n \in \mathbb{N}}\{v \in Y: g(x, v) \geq \alpha(x)-1 / n\} .
$$

So, by a classical result (see, for instance, [5], p. 170), $S^{y}$ and $T_{x}$ are connected (and non-empty, of course). Consequently, thanks to Theorem 2 (case $\left(\delta_{4}\right)$ ), one has $S \cap T \neq \emptyset$. Plainly, any point in $S \cap T$ satisfies our conclusion.

Remark 4. Compare Theorem 10 with Theorem 4 of [3]. 
The next result, suggested by the new approach recently proposed in [7], is about the existence of zeros for certain operators.

THEOREM 11. Let $V$ be a topological space, $X$ a real topological vector space (with topological dual $X^{*}$ ), and $\Phi: V \rightarrow X^{*}$ an operator such that the set $\{x \in X: v \rightarrow\langle\Phi(v), x\rangle$ is continuous $\}$ is dense in $X$. Assume that there are a continuous function $u:[0,1] \rightarrow V$, a continuous function $\alpha:[0,1] \rightarrow \mathbb{R}$, a lower semicontinuous function $f: X \rightarrow[0,1]$ and an upper semicontinuous function $g: X \rightarrow[0,1]$, with $f(x) \leq g(x)$ for all $x \in X$, such that $\langle\Phi(u(y)), x\rangle \neq \alpha(y)$ for every $(x, y) \in X \times[0,1]$ satisfying $y \in[f(x), g(x)]$. Then the operator $\Phi$ vanishes at some point of $V$.

Proof. Put

$$
S=\{(x, y) \in X \times[0,1]:\langle\Phi(u(y)), x\rangle=\alpha(y)\}
$$

and

$$
T=\{(x, y) \in X \times[0,1]: y \in[f(x), g(x)]\} .
$$

Arguing by contradiction, assume that $\Phi(v) \neq 0$ for all $v \in V$. In particular, this implies that $p_{[0,1]}(S)=[0,1]$. Also, observe that $T$ is closed and $S \cap T=\emptyset$. Then, in view of Theorem 1, $S$ must be disconnected. At this point, we can apply Theorem 1 and Proposition 1 of [7] to the operator $\Phi \circ u$, and so $\Phi(u(y))=0$ for some $y \in[0,1]$, a contradiction.

We conclude with an application of Theorem 1 to compact mappings in Banach spaces. First, we need the following

Proposition 5. Let $X$ be a topological space, $Y \subseteq \mathbb{R}$ a compact interval, and $f: X \times Y \rightarrow \mathbb{R}$ an upper semicontinuous function such that $f(\cdot, y)$ is continuous for each $y \in Y$. Moreover, let $\lambda \in \mathbb{R}$ be such that

$$
\{y \in Y: f(x, y)>\lambda\} \neq \emptyset
$$

and

$$
\inf \{y \in Y: f(x, y) \geq \lambda\}=\inf \{y \in Y: f(x, y)>\lambda\}
$$

for each $x \in X$. Then the function $x \rightarrow \inf \{y \in Y: f(x, y) \geq \lambda\}$ is continuous.

Proof. For each $x \in X$, put

$$
F(x)=\{y \in Y: f(x, y) \geq \lambda\} \quad \text { and } \quad G(x)=\{y \in Y: f(x, y)>\lambda\} .
$$

Our assumptions imply that the multifunction $F$ is upper semicontinuous ([4], Theorem 7.1.16) and that the multifunction $G$ is lower semicontinuous (in fact, its fibers are open). Consequently, the multifunction $x \rightarrow[\inf F(x), \sup F(x)]$ is upper semicontinuous and the multifunction $x \rightarrow[\inf G(x), \sup G(x)]$ is lower semicontinuous ([4], Theorem 7.3.17). This readily implies that the function 
$x \rightarrow \inf F(x)$ (resp. $x \rightarrow \sup F(x)$ ) is lower (resp. upper) semicontinuous and that the function $x \rightarrow \inf G(x)$ (resp. $x \rightarrow \sup G(x)$ ) is upper (resp. lower) semicontinuous. The proof is complete.

REMARK 5. It is clear from the proof that Proposition 5 is still true replacing, in the assumptions and in the conclusion, "inf" by "sup".

TheOREM 12. Let $E$ be a Banach space, $[a, b]$ a compact real interval, $\Omega$ a non-empty open bounded subset of $E$, and $f$ a continuous function from $\bar{\Omega} \times$ $[a, b]$ into $E$, with relatively compact range. Assume that $f(x, y) \neq x$ for all $(x, y) \in \partial \Omega \times[a, b]$ and that the Leray-Schauder index of $f(\cdot, a)$ is not zero. Then, for every lower semicontinuous function $\varphi: \Omega \rightarrow[a, b]$ and every upper semicontinuous function $\psi: \Omega \rightarrow[a, b]$ with $\varphi(x) \leq \psi(x)$ for all $x \in \Omega$, there exist $x^{*} \in \Omega$ and $y^{*} \in\left[\varphi\left(x^{*}\right), \psi\left(x^{*}\right)\right]$ such that $f\left(x^{*}, y^{*}\right)=x^{*}$.

In addition, if for some sequence $\left\{\lambda_{n}\right\}$ of positive real numbers with $\inf _{n \in \mathbb{N}} \lambda_{n}$ $=0$, one has

$$
\inf \left\{y \in[a, b]:\|f(x, y)-x\| \geq \lambda_{n}\right\}=\inf \left\{y \in[a, b]:\|f(x, y)-x\|>\lambda_{n}\right\}
$$

for each $x \in \Omega$ and $n \in \mathbb{N}$ for which

$$
\left\{y \in[a, b]:\|f(x, y)-x\|>\lambda_{n}\right\} \neq \emptyset,
$$

then there exists $x_{0} \in \Omega$ such that $f\left(x_{0}, y\right)=x_{0}$ for all $y \in[a, b]$.

Proof. Thanks to the classical Leray-Schauder continuation principle (see, for instance, [8], Theorem 14.C), there exists a compact connected set $S \subseteq$ $\Omega \times[a, b]$ such that $p_{[a, b]}(S)=[a, b]$ and $f(x, y)=x$ for all $(x, y) \in S$. Let $\varphi, \psi$ be as in the statement. Put

$$
T=\{(x, y) \in \Omega \times[a, b]: y \in[\varphi(x), \psi(x)]\} .
$$

Then, in view of Theorem 1 , one has $S \cap T \neq \emptyset$, which yields the first conclusion of the theorem.

Now, assume that there is some $\left\{\lambda_{n}\right\}$ as in the statement. For each $n \in \mathbb{N}$, put

$$
V_{n}=\left\{(x, y) \in \Omega \times[a, b]:\|f(x, y)-x\|>\lambda_{n}\right\} .
$$

Observe that $p_{\Omega}\left(V_{n}\right) \neq \Omega$. Indeed, if $p_{\Omega}\left(V_{n}\right)=\Omega$, then in view of Proposition 5 , the function $x \rightarrow \inf \left\{y \in[a, b]:\|f(x, y)-x\| \geq \lambda_{n}\right\}$ would be continuous in $\Omega$, and so, by Theorem 1 again, its graph should meet $S$, which is clearly absurd. Then pick $x_{n} \in \Omega$ such that $\left\|f\left(x_{n}, y\right)-x_{n}\right\| \leq \lambda_{n}$ for all $y \in[a, b]$. Since $f(\bar{\Omega} \times[a, b])$ is relatively compact and $\inf _{n \in \mathbb{N}} \lambda_{n}=0$, the sequence $\left\{x_{n}\right\}$ admits some convergent subsequence. Plainly, the limit of such a subsequence satisfies the second conclusion of the theorem. 


\section{REFERENCES}

[1] K. Deimling, Multivalued Differential Equations, Walter de Gruyter, 1992.

[2] R. Engelking, Dimension Theory, North-Holland, 1978.

[3] K. FAN, Applications of a theorem concerning sets with convex sections, Math. Ann. 163 (1966), 189-203.

[4] E. Klein and A. C. Thompson, Theory of Correspondences, Wiley, 1984.

[5] K. Kuratowski, Topology, vol. II, Academic Press, 1968.

[6] B. RICCERI, Some topological mini-max theorems via an alternative principle for multifunctions, Arch. Math. (Basel) 60 (1993), 367-377.

[7] Existence of zeros via disconnectedness, J. Convex Anal. (to appear).

[8] E. Zeidler, Nonlinear Functional Analysis, vol. I, Springer-Verlag, 1986.

Manuscript received February 3, 1995

Biagio RicceRI

Department of Mathematics

University of Catania

Viale A. Doria 6

95125 Catania, ITALY

TMNA : Volume $5-1995-\mathrm{N}^{\mathrm{o}} 2$ 